\title{
General Concept of Quantization
}

\author{
F. A. Berezin^ \\ Academy of Sciences of the Ukrainian SSR, Institute for Theoretical Physics, \\ Kiev, Ukrainian, SSR
}

Received June 15, 1974

\begin{abstract}
The general definition of quantization is proposed. As an example two classical systems are considered. For the first of them the phase space is a Lobachevskii plane, for the second one the two-dimensional sphere.
\end{abstract}

It is generally accepted that the quantization is an algorithm by means of which a quantum system corresponds to a classical dynamic one. Furthermore, it is required that in the limit $h \rightarrow 0$ where $h$ is the Planck's constant, a quantum dynamic system change to a corresponding classical one. This requirement is called the correspondence principle. It is quite obvious that there exist quite a lot quantizations obeying the correspondence principle; the quantum description of a physical phenomenon is more detailed than the classical one, and so there are certain phenomena the difference between which is displayed in their quantum description, whereas their classical description does not show this difference.

The following intuitive method of quantizing the classical dynamic systems with a flat phase space has become well known since the Schrödinger equation was first written down. If a system has $n$ degrees of freedom, its phase space is a real linear space $\mathscr{R}^{2 n}$ of dimension $2 n$, and the observables are the functions $f(p, q), p, q \in \mathscr{R}^{2 n}, p=\left(p_{1} \ldots p_{n}\right)$, $q=\left(q_{1} \ldots q_{n}\right)$, where $p_{i}, q_{i}$ are the momenta and coordinates. The Hilbert space of states of a corresponding quantum system is a space of functions $f(x), x=\left(x_{1}, \ldots, x_{n}\right)$ of $n$ real variables with a summable square. The operators in $L^{2}\left(\mathscr{R}^{n}\right) \hat{p}_{k}, \hat{q}_{k}$ are compared to the classical momenta and coordinates, $p_{k}, q_{k}$ using the formulas

$$
\left(\hat{q}_{K} f\right)(x)=x_{K} f(x), \quad\left(\hat{p}_{K} f\right)(x)=\frac{h}{i} \frac{\partial f}{\partial x_{K}} .
$$

A "quantum observable" - the operator $f(\hat{p}, \hat{q})$ obtained by "replacing the real variables $p_{i}, q_{i}$ by the operators $\hat{p}_{i}, \hat{q}_{i}$ in $f(p, q)$ " corresponds to an arbitrary observable $f(p, q)$. However, the operators $\hat{p}_{i}, \hat{q}_{i}$ do not commute, and so this algorithm is applicable, provided that the analytical expression for $f$ contains no products such as $p_{i} q_{i}$. For the case of arbitrary $f(p, q)$ the algorithm in question should be specified. One of such specifications which posseses a number of remarkable features is due to Weyl [1].

$\star$ The Moscow State University. 
In a case when the phase of a classical dynamic system is not plane it contains no natural coordinates $p_{i}, q_{i}$ and so all the quantization methods based on the special features of these coordinates prove inconsistent. The mechanical systems whose phase space is not plane seem to be more frequent than they are thought to be. The simplest example is a solidstate body with a fixed point (here a two-dimensional sphere serves as a phase space). The topicality of such systems in field theory has recently become increasingly apparent.

This paper is aimed at defining the mathematical nature of quantization algorithms. A general definition of quantization of an arbitrary mechanical system is proposed. Then this general definition is exemplified by the systems with one degree of freedom for which the phase space is a Lobachevskii plane or a sphere.

\section{§ 1. Classical Mechanics}

1. Definition. In a general case the classical mechanics will be defined as a pair $(\mathfrak{M}, \omega)$ where $\mathfrak{M}$ is some differentiable manifold and $\omega$ is a skew-symmetric tensor field on this manifold. The field $\omega$ in local coordinates should have components $\omega^{i j}(x)$ satisfying the condition ${ }^{1}$

$$
\omega^{\gamma k} \frac{\partial \omega^{\alpha \beta}}{\partial x^{k}}+\omega^{\beta k} \frac{\partial \omega^{\gamma \alpha}}{\partial x^{k}}+\omega^{\alpha k} \frac{\partial \omega^{\beta \gamma}}{\partial x^{k}}=0 .
$$

Let us denote by $\mathscr{A}(\mathfrak{M})$ a set of differentiable functions on $\mathfrak{M} . \mathscr{A}(\mathfrak{M})$ is a commutative and associative algebra with respect to the standard addition and multiplication, and is a Lie algebra with respect to the Poisson bracket

$$
[f, g]=\omega^{i j} \frac{\partial f}{\partial x^{i}} \frac{\partial g_{i}}{\partial x^{j}} .
$$

The fact that the Poisson bracket (1.2) defines the Lie algebra, i.e. that the Yacobi identity

$$
\left[f_{1},\left[f_{2}, f_{3}\right]\right]+\left[f_{3},\left[f_{1}, f_{2}\right]\right]+\left[f_{2},\left[f_{3}, f_{1}\right]\right]=0
$$

is valid, is equivalent to the Condition (1.1). This is easily verified by direct calculations. Furthermore, from this it also follows that the Condition (1.1) is independent of the choice of coordinates.

In a case when $\operatorname{det}\left\|\omega^{i j}\right\| \neq 0$ there exists an inverse matrix $\left\|\omega_{i j}(x)\right\|$ for every $x \in \mathfrak{M}$. The Condition (1.1) is written in terms of the matrix elements as $\omega_{i j}$

$$
\frac{\partial \omega_{i j}}{\partial x^{k}}+\frac{\partial \omega_{k i}}{\partial x^{j}}+\frac{\partial \omega_{j k}}{\partial x^{i}}=0
$$

The Condition (1.4) implies that the external form $\omega=\omega_{i j} d x^{i} \wedge d x^{j}$ is closed. Conversely, if a nondegenerate external form $\omega=\omega_{i j} d x^{i} \wedge d x^{j}$

1 Tensor notation is meant here and in similar situations in what follows. In particular, the double twice repeated index stands for summation. 
is given on the manifold $\mathfrak{M}$ then its components $\omega_{i j}$ satisfy the Relation (1.4), and the inverse-matrix components satisfy the Relation (1.1). Consequently, in this case $(\mathfrak{M}, \omega)$ where $\omega$ is the tensor field with components $\omega^{i j}$, is a classical mechanics.

2. Mapping of Classical Mechanics. Let $\left(\mathfrak{M}_{1}, \omega_{1}\right),\left(\mathfrak{M}_{2}, \omega_{2}\right)$ be classical mechanics with nondegenerate tensor fields $\omega_{1}^{\alpha \beta}, \omega_{2}^{\alpha \beta}, \omega_{1}, \omega_{2}$ are the corresponding external forms. The diffeomorphism $\mathfrak{M}_{1} \rightarrow \mathfrak{M}_{2}$ mapping $\omega_{1}$ into $\omega_{2}$ will be called a mapping $\varphi:\left(\mathfrak{M}_{1}, \omega_{1}\right) \rightarrow\left(\mathfrak{M}_{2}, \omega_{2}\right)$. More specifically if $y=\varphi(x) \in \mathfrak{M}_{2}, y^{i}=\varphi^{i}(x)$ are the local coordinates of the point $y$ and $x^{i}$ the local coordinates of the point $x$, then

$$
\omega_{1, \alpha \beta}(x)=\omega_{2, i j} \frac{\partial y^{i}}{\partial x^{\alpha}} \frac{\partial y^{j}}{\partial x^{\beta}} .
$$

3. Examples. 1) $\mathfrak{M}=\mathscr{R}^{2}$ is a plane with coordinates $p, q$. $\omega^{12}=-\omega^{21}=1$,

$$
[f, g]=\frac{\partial f}{\partial p} \frac{\partial g}{\partial q}-\frac{\partial f}{\partial q} \frac{\partial g}{\partial p}
$$

2) $\mathfrak{M}=K^{1} \times \mathscr{R}^{1}-$ is a two-dimensional cylinder. The coordinates will be denoted by $p, q$. Moreover, $p \in \mathscr{R}^{1}, q \in K^{1}$ i.e. $p$ is an arbitrary real number, $-\infty<p<\infty, q$ is the point of a circumference, $0 \leqq q<2 \pi$. The differentiable functions on $\mathfrak{M}$ are periodic in $q$ with period $2 \pi$. The tensor $\omega$ and the Poisson bracket have the previous form.

3) $\mathfrak{M}=K^{1} \times K^{1}$ is a two-dimensional torus. The coordinates $p, q$ independently run over the circumference, $0 \leqq p<2 \pi$. $0 \leqq q<2 \pi$. The differentiable functions on $\mathfrak{M}$ are periodic in each of the variables with period $2 \pi$. The Poisson bracket has the form (1.5).

4) $\mathfrak{M}=S^{2}$ is a two-dimensional sphere. The measure on $S$ invariant under rotations has the form $d \mu=r^{2} \sin \theta d \varphi \wedge d \theta$, where $r$ is the radius of the sphere,

The same expression serves as the 2-form required. Therefore the Poisson bracket in polar coordinates has the form

$$
[f, g]=\frac{1}{r^{2} \sin \theta}\left(\frac{\partial f}{\partial \varphi} \frac{\partial g}{\partial \theta}-\frac{\partial f}{\partial \theta} \frac{\partial g}{\partial \varphi}\right)
$$

In what follows it is more convenient to use complex coordinates instead of the polar ones on $S^{2}$.

These coordinates are introduced using the stereographic projection:

$$
|z|=2 r \operatorname{ctg} \frac{\theta}{2}, \quad \arg z=\varphi .
$$

The external form $\omega$ in these coordinates is

$$
\omega=\frac{1}{2 i}\left(1+\frac{z \bar{z}}{4 r^{2}}\right)^{-2} d z \wedge d \bar{z}
$$


and the Poisson bracket has form

$$
[f, g]=2 i\left(1+\frac{z \bar{z}}{4 r^{2}}\right)^{2}\left(\frac{\partial f}{\partial z} \frac{\partial g}{\partial \bar{z}}-\frac{\partial f}{\partial \bar{z}} \frac{\partial g}{\partial \bar{z}}\right) .
$$

5) $\mathfrak{M}=\mathscr{L}^{2}$ is a Lobachevskii plane. We use the Poincare model for the Lobachevskii plane: the Lobachevskii plane will be identified with a circle (of radius $2 r$ and with its centre at zero) in a complex plane. On the Lobachevskii plane there is an external form $\omega$ invariant under movements. This form is an element of an invariant volume

$$
\omega=\frac{1}{2 i}\left(1-\frac{z \bar{z}}{4 r^{2}}\right)^{2} d z \wedge d \bar{z}
$$

The corresponding Poisson bracket has the form

$$
[f, g]=2 i\left(1-\frac{z \bar{z}}{4 r^{2}}\right)^{2}\left(\frac{\partial f}{\partial z} \frac{\partial g}{\partial \bar{z}}-\frac{\partial f}{\partial \bar{z}} \frac{\partial g}{\partial z}\right) .
$$

6) Let $G$ be an arbitrary Lie algebra, $C_{k}^{i j}$ its structural constants, $i, j, k=1, \ldots, v$. As $\mathfrak{M}$ we consider a $v$-dimensional Euclidean space $\mathscr{R}^{v}$ conjugate of $G$. We set

$$
\omega^{i j}=C_{k}^{i j} x^{k}
$$

The Property (1.1) follows from the Yacobi identity for $G$. This example is closely associated with Examples 1), 4), and 5). Example 6 was considered previously in [2].

$6_{1}$ ) As $G$ we consider the Heisenberg-Weyl algebra. Let $e_{1}, e_{2}, e_{0}$ be a basis in $G$, with the standard relations

$$
\left[e_{1}, e_{2}\right]=e_{0}, \quad\left[e_{1}, e_{0}\right]=\left[e_{2}, e_{0}\right]=0 .
$$

The coordinates in $\mathscr{R}^{3}$, corresponding to $e_{0}, e_{1}, e_{2}$, will be denoted by $\varepsilon, p, q$. In this case $\omega^{12}=-\omega^{21}=\varepsilon$, and the Poisson bracket has the classical form:

$$
[f, g]=\varepsilon\left(\frac{\partial f}{\partial p} \frac{\partial g}{\partial q}-\frac{\partial f}{\partial q} \frac{\partial g}{\partial p}\right)
$$

$\left.6_{2}\right) G$ is a Lie algebra of group $S O(3)$. If in $G$ one chooses a standard basis $e_{1}, e_{2}, e_{3}$, with the relations $\left[e_{1}, e_{2}\right]=e_{3},\left[e_{3}, e_{1}\right]=e_{2},\left[e_{2}, e_{3}\right]=e_{1}$, then the Poisson bracket takes on the form

$$
[f, g]=\left|\begin{array}{lll}
x^{1} & x^{2} & x^{3} \\
\frac{\partial f}{\partial x^{1}} & \frac{\partial f}{\partial x^{2}} & \frac{\partial f}{\partial x^{3}} \\
\frac{\partial g}{\partial x^{1}} & \frac{\partial g}{\partial x^{2}} & \frac{\partial g}{\partial x^{3}}
\end{array}\right|
$$


Let us introduce the polar coordinates $x^{1}=r \sin \theta \cos \varphi, x^{2}=r$ - $\sin \theta \sin \varphi, x^{3}=r \cos \theta$. On replacing the variables, we obtain from (1.12):

$$
[f, g]=\frac{1}{r \sin \theta}\left(\frac{\partial f}{\partial \theta} \frac{\partial g}{\partial \varphi}-\frac{\partial f}{\partial \varphi} \frac{\partial g}{\partial \theta}\right) .
$$

Thus, for the functions $f$ at fixed $r$ the Poisson brackets (1.12) transforms, with an accuracy up to a multiplier, to (1.6).

In a similar way the Poisson brackets are connected on the Lie algebra of a three-dimensional Lorentz group, and on the Lobachevskii plane.

\section{§ 2. Quantization}

1. General Definition. The associative algebra $\mathfrak{A}$ with involution, possessing the properties to be given below, is identified as the quantization of a classical mechanics $(\mathfrak{M}, \omega)$. that

1) There is a family $A_{h}$ of associative algebras with involution, such

$1_{1}$ ) the index $h$ runs through the set $E$ on the positive side of the real axis, where 0 is a limit point ( 0 is not involved in $E)$.

$1_{2}$ ) The algebra $\mathfrak{A}$ consists of the functions $f(h)$ taking values in $A_{h}$. The involution and multiplication in $\mathfrak{A}$ are connected with the involution and multiplication in $A_{h}$ in a usual way: $\left(f^{\odot}\right)(h)=(f(h))^{6}$ where $\odot$, $\sigma$ are the involutions in $\mathfrak{A}$ and $A_{h}$, respectively, $\left(f_{1} \circledast f_{2}\right)(h)=f_{1}(h) * f_{2}(h)$, where $\circledast, *$ are the multiplications in $\mathfrak{A}$ and $A_{h}$ respectively. In the remainder of the paper the involution and multiplication in algebras $\mathfrak{A}$ and $A_{h}$ are denoted by the same symbols.

2) There exists a homomorphism $\varphi$ of algebra $\mathfrak{A}$ into algebra $\mathscr{A}(\mathfrak{M})$ of the differentiable functions on $\mathfrak{M}$ with standard operations of addition and multiplication. The homomorphism should have the following properties

i) for the any two points $x_{1}, x_{2} \in \mathfrak{M}$ there is a function $f(x) \in \varphi(\mathfrak{A})$ such that $f\left(x_{1}\right) \neq f\left(x_{2}\right)$,

ii) $\varphi\left(\frac{1}{h}(f * g-g * f)\right)=i\left[\varphi\left(f_{1}\right), \varphi\left(f_{2}\right)\right], \quad i=\sqrt{-1}$,

where $*$ denotes the multiplication in $\mathfrak{A}$, and $[\cdot, \cdot]$ denotes the Poisson bracket in $\mathscr{A}(\mathfrak{M})$,

iii)

$$
\varphi\left(f^{\sigma}\right)=\overline{\varphi(f)},
$$

where $f \rightarrow f^{\sigma}$ stands for the involution in $\mathfrak{A}$, and the line for a complex conjugation.

2. Special Quantization. By this quantization is meant one which has two additional properties.

3) Algebra $A_{h}$ consists of the differentiable functions $f(x), x \in \mathfrak{M}$.

4) Algebra $\mathfrak{A}$ consists of the functions $f(h, x), f(h, x) \in A_{h}$ for fixed $h$. 
5) Homomorphism $\varphi: \mathfrak{U} \rightarrow \mathscr{A}(\mathfrak{M})$ is given by

$$
\varphi(f)=\lim _{h \rightarrow 0} f(h, x) .
$$

A consistent theory is now available only for special quantizations $[3,4]^{2}$. All the special quantizations hitherto investigated also posses the following properties.

6) Algebra $A_{h}$ has unity, and here the function $f_{0}(h, x) \equiv 1$ serves as this unity.

7) Algebra $A_{h}$ is an algebra with a trace. In this case

$$
\operatorname{Sp} f=c \int f(x) d \mu(x)
$$

where $d \mu(x)$ is some measure on $\mathfrak{M}$ and $c$ is an indefinite multiplier ${ }^{3}$.

Note that if the tensor field $\omega^{i j}(x)$ is not degenerate, i.e. if on $\mathfrak{M}$ there exists an external closed form of the second degree $\omega=\omega_{i j} d x^{i} \wedge d x^{j}$ then on $\mathfrak{M}$ there exists a natural measure

$$
d \mu(x)=c \omega^{\frac{n}{2}} .
$$

3. Functor of Quantization. Let $\mathfrak{B}_{1}, \mathfrak{B}_{2}$ be algebras constructed in analogy to quantizations: there exist algebras $B_{h}^{(1)}, h \in E_{1}, B_{h}^{(2)}, h \in E_{2}$ such that $\mathfrak{B}_{i}$ consist of the functions $f(h), h \in E_{i}$ taking values in $B_{h}^{(i)}$. The homomorphism $\psi: \mathfrak{B}_{1} \rightarrow \mathfrak{B}_{2}$ of algebras with such structure is called admissible, if it is generated by homomorphisms $\psi_{h}$ of algebras $B_{h}^{(i)}:(\psi f)(h)=\psi_{h} f(h)$.

(In order for the admissible homorphism $\mathfrak{B}_{1} \rightarrow \mathfrak{B}_{2}$ to exist it is necessary that $E_{1} \subset E_{2}$.)

The admissible isomorphism of algebras $\mathfrak{B}_{i}$ is defined in a similar manner.

We fix the set of classical mechanics $\mathscr{E}$ and the category $\mathscr{K}$ of the mappings of the elements of $\mathscr{E}$. Let the quantization $\mathfrak{U}$ correspond to every classical mechanics $(\mathfrak{M}, \omega) \in \mathscr{E}$.

The correspondence $(\mathfrak{M}, \omega) \leadsto \mathfrak{U}$ will be referred to as the functor of quantization, if for any pair of classical mechanics $\left(\mathfrak{M}_{i}, \omega_{i}\right) \in \mathscr{E}, i=1,2$ related by $\left(\mathfrak{M}_{2}, \omega_{2}\right)=\phi\left(\mathfrak{M}_{1}, \omega_{1}\right), \phi \in \mathscr{K}$, there exists an admissible homomorphism $\phi$ such that the diagram

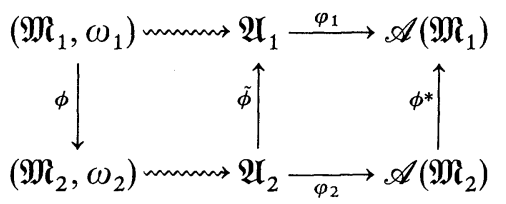

${ }^{2}$ References [7] gives two separate examples of quantizations which are not special. Manifold $\mathfrak{M}$ in the first case is a two-dimensional cylinder, and in the second a twodimensional torus. In natural local coordinates the Poisson bracket in both the cases has the form (1.5).

3 The linear functional $\operatorname{Sp} f$ defined on some subset $\tilde{A}_{h} \subset A_{h}$ is termed a trace, if the sets $A_{h}$ and $\operatorname{Sp} f$ have the properties 1) if $f_{2} * f_{2} \in \tilde{A}_{h}$, then $\left.f_{2} * f_{1} \in \tilde{A}_{1}, 2\right)$ if $f_{1} * f_{2} \in \tilde{A}_{h}$, then $\operatorname{Sp}\left(f_{1} * f_{2}\right)=\operatorname{Sp}\left(f_{2} * f_{1}\right)$. It is clear that the functional $\operatorname{Sp} f$ is defined with an accuracy up to a multiplier. 
is commutative. Here $\varphi_{1}, \varphi_{2}$ are the homomorphisms used in defining the quantization and $\phi^{*}$ is the mapping of the functions, which is conjugate of the diffeomorphism $\phi$.

The functor of quantization will be denoted by $Q$. If the correspondence $(\mathfrak{M}, \omega) \leadsto \mathfrak{U}$ is a functor, we write $\mathfrak{U}=Q(\mathfrak{M}, \omega)$ in order to emphasize this point.

The quantization functor $Q$ is termed special, if all the quantizations $\mathfrak{U}=Q(\mathfrak{M}, \omega)$ are special.

4. Naturalness. For objects concerned with special quantizations there is an important concept of naturalness. Let $\mathscr{K}$ be some category of mappings of classical mechanics, $\left(\mathfrak{M}_{i}, \omega_{i}\right), \mathfrak{U}_{i}, i=1,2$ the classical mechanics and their quantizations.

The admissible homomorphism $\psi: \mathfrak{A}_{1} \rightarrow \mathfrak{U}_{2}$ is called natural with respect to $\mathscr{K}$, if exists a mapping $\phi \in \mathscr{K}, \phi\left(\mathfrak{M}_{2}, \omega_{2}\right)=\left(\mathfrak{M}_{1}, \omega_{1}\right)$ such that

$$
(\psi f)(h, x)=f(h, \varphi(x)), \quad x \in \mathfrak{M}_{2} .
$$

The Relation (2.3) implies that at fixed $h$ the homomorphism $\psi$ is the mapping of the functions, conjugate of the diffeomorphism $\varphi$. Therefore, using not too strict notations we write $\psi=\varphi^{*}$.

The special quantization functor $Q$ is termed natural with respect to $\mathscr{K}$, if $\tilde{\varphi}=\varphi^{*}$ in the Diagram (2.2).

The special quantization $\mathfrak{A}=Q(\mathfrak{M}, \omega)$ where $Q$ is the special functor, is reffered to as natural with respect to the category $\mathscr{K}$, if the functor $Q$ has this property.

Remark. One can show (see [7]) that there is no quantization functor $Q$ natural with respect to the category of all the mappings of classical mechanics.

5. Groups of Motion. Let $(\mathfrak{M}, \omega)$ be a classical mechanics and $G$ a transformation group $\mathfrak{M}$ contained in some category $\mathscr{K}$ of the mappings of classical mechanics. The latter circumstance implies, in particular, that the transformation $\tau_{g}$ in $\mathscr{A}(\mathfrak{M})$ :

$$
\left(\tau_{g} f\right)(x)=f(g x)
$$

is an automorphism of the Lie algebra of Poisson brackets.

Let $\mathfrak{U}$ be the quantization of the mechanics $(\mathfrak{M}, \omega)$ natural with respect to $\mathscr{K}$. From the above definitions it follows immediately, that the Transformation (2.4) is the automorphism of all algebras $A_{h}$.

6. Equivalency of Quantizations. Let $\mathfrak{U}$ be quantization of the classical mechanics $(\mathfrak{M}, \omega), E$ a set of the values $h, S$ some one-to-one transformation of $E$. The mapping $S$ generates automorphism $S^{*}$ of the algebra $\mathfrak{U}$ :

$$
\left(S^{*} f\right)(h)=f(s h) .
$$

Now let $\mathfrak{U}_{1}, \mathfrak{U}_{2}$ be the quantizations of the same classical mechanics $(\mathfrak{M}, \omega)$. The quantizations $\mathfrak{A}_{1}$ and $\mathfrak{A}_{2}$ are called equivalent, if

1) there is an isomorphism $\phi: \mathfrak{U}_{1} \rightarrow \mathfrak{U}_{2}$ and an isomorphism $S^{*}: \mathfrak{U}_{1} \rightarrow \mathfrak{U}_{1}$ (of the type mentioned above) such that the isomorphism 
$\phi S^{*}: \mathfrak{A}_{1} \rightarrow \mathfrak{U}_{2}$ is admissible, 2) the diagram

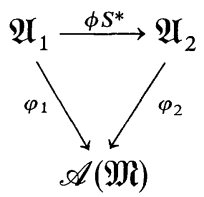

is commutative. Here $\varphi_{i}$ are the homomorphisms used in defining the quantization.

In a case when on $\mathfrak{M}$ acts the transformation group $G$, contained in the category with respect to which the quantizations $\mathfrak{U}_{1}$ and $\mathfrak{A}_{2}$ are natural, the notion of a natural equivalency is introduced. In addition to $(2.5)$, the diagram

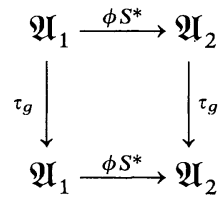

should also be commutative. Here $\tau_{q}$ is an isomorphism of the type (2.4).

\section{§ 3. Supercomplete Systems of Vectors ${ }^{4}$}

At present the quantization is constructed for classical mechanics for which the phase spaces are so-called complex symmetric spaces. They include the Lobachevskii plane and a two-dimensional sphere. In all these cases the quantization is constructed by a general method which will be presented here in an abstract form.

1. Main Definition. Let $H$ be a Hilbert space and $M$ some set with measure $d \alpha$. The system of vectors $e_{\alpha} \in H, \alpha \in M$ is called supercomplete, if for any $f, g \in H$ there holds the Parseval identity

$$
(f, g)=\int\left(f, e_{\alpha}\right)\left(e_{\alpha}, g\right) d \alpha .
$$

Let us denote by $d \mu(\alpha)$ a measure absolutely continuous in $d \alpha$

$$
d \mu(\alpha)=\left(e_{\alpha}, e_{\alpha}\right) d \alpha .
$$

Note that a supercomplete system generates the imbedding of $H$ into the space $L^{2}(M)$ according to the formula

$$
f \mapsto f(\alpha)=\left(f, e_{\alpha}\right) .
$$

From now on we assume that the space $H$ is realized as the subspace $L^{2}(M)$.

From (3.2) it follows, in particular, that

$$
e_{\alpha}(\beta)=\left(e_{\alpha}, e_{\beta}\right)=\overline{\left(e_{\beta}, e_{\alpha}\right)}=\overline{e_{\beta}(\alpha)} .
$$

\footnotetext{
${ }^{4}$ In this section the contents of Ref. [5] is given in brief.
} 
Let $\hat{P}_{\alpha}$ be an orthogonal projector on $e_{\alpha}$. The function

$$
A(\alpha)=\operatorname{Sp}\left(\hat{A} \hat{P}_{\alpha}\right)=\frac{\left(\hat{A} e_{\alpha}, e_{\alpha}\right)}{\left(e_{\alpha}, e_{\alpha}\right)}
$$

is called a covariant symbol of the operator $\hat{A}$ in $H_{0}$. The function $\AA(\alpha)$ with the use of which the operator $\hat{A}$ is representable as

$$
\hat{A}=\int \stackrel{\circ}{A}(\alpha) \hat{P}_{\alpha} d \mu(\alpha)
$$

is called a contravariant symbol of the operator $\hat{A}$.

The definitions show that the covariant symbol is uniquely defined for any operator whose domain contains $e_{\alpha}$ for all $\alpha \in M$, in particular, for any bounded operator.

It might be that various operators have the same covariant symbol. Below we shall see that this is not so in the cases of interest: there is a one-to-one correspondence between the operators and the covariant symbols. As regards the contravariant symbols, they are defined, in general, not for all bounded operators and not always uniquely, but the operator can always be uniquely reconstructed from the contravariant symbol.

Note that (3.5) yields an important interpretation of contravariant symbols. Let us denote by $\hat{B}$ an operator in $L^{2}(M)$ such

$$
(\hat{B} f)(\alpha)=\stackrel{\circ}{(\alpha)} f(\alpha)
$$

and by $T$ the orthogonal projector from $L^{2}(M)$ on $H$. The formula (3.5) is equivalent to

$$
A=T \hat{B} T .
$$

The importance of co- and contravariant symbols being considered simultaneously consists in the fact that they can be used to obtain the estimates of upper and lower bounds for the spectral characteristics of the operator $\hat{A}$. We give the most important relevant results.

1) Let $\mathscr{D}(\hat{A})$ be a Hopf set of the operator $\hat{A}$, i.e. the set of a complex plane, consisting of all numbers such as $(\hat{A} \hat{f}, f),\|f\|=1, \mathscr{D}(A)$ the set of the values of the covariant symbol $A$ and $\mathscr{D}(A)$ a convex hull of the set of values of the contravariant symbol $\AA$. The inclusions

$$
\mathscr{D}(A) \subset \mathscr{D}(\hat{A}) \subset \mathscr{D}(\stackrel{\AA}{)}
$$

are valid.

In a case when the operator $\hat{A}$ is self-adjoint, (3.7) yields in particular, the estimate of its spectrum (the covariant symbol of a self-adjoint

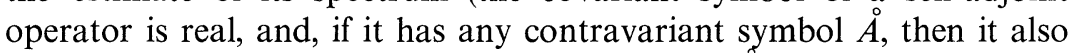
has a real contravariant symbol which is $\operatorname{Re} A$ ). For a self-adjoint operator, it also follows from (3.7) that

$$
\operatorname{Sup}|A(\alpha)| \leqq\|A\| \leqq \operatorname{Sup}|\AA(\alpha)| .
$$


2) Let $\varphi(t)$ be a convex (downward) function of a real variable. Then

$$
\int \varphi(A(\alpha)) d \mu(\alpha) \leqq \operatorname{Sp} \varphi(\hat{A}) \leqq \int \varphi(\AA(\alpha)) d \mu(\alpha) .
$$

In a case when the operator $\hat{A}$ is semi-bounded from below, the convergence of the integral on the right-hand side of (3.8) implies the existence of $\operatorname{Sp} \varphi(\hat{A})$, and the existence of $\operatorname{Sp} \varphi(\hat{A})$ implies the convergence of the integrals on the left-hand side of (3.8).

3) If the contravariant symbol of a self-adjoint operator is semibounded from below, then

$$
e^{-t \hat{A}}=\lim _{n \rightarrow \infty}\left(T e^{-\frac{t}{n} \hat{B}} T\right)^{n}
$$

where $B$ and $T$ are the same operators as in (3.6).

2. Algebra of Covariant Symbols. Let $\hat{A}$ be a bounded operator in $H$, and $A(\alpha)$ its covariant symbol. We show that the action of the operator on the vector defined by the formula

where

$$
(\hat{A} f)(\alpha)=\int f(\beta) A(\beta, \alpha) \frac{\left(e_{\beta}, e_{\alpha}\right)}{\left(e_{\beta}, e_{\beta}\right)} d \mu(\beta)
$$

$$
A(\alpha, \beta)=\frac{\left(\hat{A} e_{\alpha}, e_{\beta}\right)}{\left(e_{\alpha}, e_{\beta}\right)}
$$

is the continuation of the function $A(\alpha)$ to $M \times M$.

Using repeatedly (3.1), we have:

$$
\begin{aligned}
(\hat{A} f)(\alpha) & =\left(\hat{A} f, e_{\alpha}\right)=\left(f, \hat{A}^{*} e_{\alpha}\right)=\int\left(f, e_{\beta}\right)\left(e_{\beta}, \hat{A}^{*} e_{\alpha}\right) d \beta \\
& =\int\left(A e_{\beta}, e_{\alpha}\right) f(\beta) d \beta=\int f(\beta) A(\beta, \alpha) \frac{\left(e_{\beta}, e_{\alpha}\right)}{\left(e_{\beta}, e_{\beta}\right)} d \mu(\beta) .
\end{aligned}
$$

It follows immediately from (3.11) that if $\hat{A}=\hat{A}_{1} \cdot \hat{A}_{2}, A, A_{1}, A_{2}$ are the covariant symbols of corresponding operators, then

$$
A(\alpha)=\int A_{1}(\gamma, \alpha) A_{2}(\alpha, \gamma) \frac{\left(e_{\alpha}, e_{\gamma}\right)\left(e_{\gamma}, e_{\alpha}\right)}{\left(e_{\alpha}, e_{\alpha}\right)\left(e_{\gamma}, e_{\gamma}\right)} d \mu(\gamma)
$$

The algebra with the multiplication law (3.12), which consists of the symbols of bounded operators, is a basis for the further construction. In what follows the manifold $\mathfrak{M}$, which serves as a phase space in classical mechanics, always plays the role of the set $M$.

It follows from the formula (3.10) that the operator $\hat{A}$ is uniquely defined by the function $A(\beta, \alpha)$ which is the continuation of $A(\alpha)$ to $M \times M$. In general, it is possible that two different functions $A_{1}(\beta, \alpha)$, $A_{2}(\beta, \alpha)$ coincide at $\beta=\alpha$, i.e. that two different operators have the same covariant symbol. In the cases of interest this is not so: the function $A(\beta, \alpha)$ turns out to be an analytic continuation of $A(\alpha)$ and thus uniquely defined from $A(\alpha)$. 
We indicate the formula connecting the co- and contravariant symbols of the same operator. The covariant symbol of the projection operator $\hat{P}_{\beta}$ on the vector $e_{\beta}$ is $\frac{\left(\hat{P}_{\beta} e_{\alpha}, e_{\alpha}\right)}{\left(e_{\alpha}, e_{\alpha}\right)}=\frac{\left(e_{\alpha}, e_{\beta}\right)\left(e_{\beta}, e_{\alpha}\right)}{\left(e_{\alpha}, e_{\alpha}\right)\left(e_{\beta}, e_{\beta}\right)}$.

It therefore follows from (3.5) that

$$
A(\alpha)=\int \AA(\beta) \frac{\left(e_{\alpha}, e_{\beta}\right)\left(e_{\beta}, e_{\alpha}\right)}{\left(e_{\alpha}, e_{\alpha}\right)\left(e_{\beta}, e_{\beta}\right)} d \mu(\beta) .
$$

The formula for a trace reads

$$
\operatorname{Sp} \hat{A}=\int A(\alpha) d \mu(\alpha)=\int \AA ⿱ 乛 ⿻ 上 丨(\alpha) d \mu(\alpha)
$$

and formula for the product trace

$$
\operatorname{Sp} \hat{A} \hat{B}=\int A(\alpha) \stackrel{\circ}{B}(\alpha) d \mu(\alpha) .
$$

\section{§ 4. Quantization on the Lobachevskii Plane}

1. The Space $\mathscr{F}_{h}$. We use the Poincare model according to which the Lobachevskii plane with a curve, which equals 2 , is identified with the unit circle $K$ in $C^{1}$. The space of analytic functions in $K$ with the scalar product

$$
(f, g)=\left(\frac{1}{h}-1\right) \int f(z) \bar{g}(z)(1-z \bar{z})^{\frac{1}{h}} d \mu(z, \bar{z}),
$$

is denoted through $\mathscr{F}_{h}$ where $d \mu(z, \bar{z})=\frac{1}{2 \pi i} \frac{d z \wedge d \bar{z}}{(1-z \bar{z})^{2}}$ is an invariant measure on the Lobachevskii plane. (The multiplier $\frac{1}{h}-1$ is connected with the normalizing condition $\left(f_{0}, f_{0}\right)=1$ for $\left.f_{0}(z) \equiv 1\right)$. Let $f_{k}$ be the orthonormalized basis in $\mathscr{F}_{h}$. Consider the function

$$
L_{h}(z, \bar{v})=\Sigma f_{k}(z) \overline{f_{k}(v)} \text {. }
$$

The series (4.2) converges absolutely and uniformly in any region such as $|z| \leqq r<1,|v| \leqq r<1$. This fact can be proved using the technique suggested by Bergman (see, for example [6]).

Consider the space $\mathscr{L}_{h}^{2}$, which consists of all measurable functions in $K$, square integrable in the measure $\left(\frac{1}{h}-1\right)(1-z \bar{z})^{\frac{1}{h}} d \mu(z, \bar{z})$. It is obvious that $\mathscr{F}_{h} \subset \mathscr{L}_{h}^{2}$ and the function $L_{h}(z, \vec{v})$ is a kernel of the orthogonal projector from $\mathscr{L}_{h}^{2}$ into $\mathscr{F}_{h}$. Therefore, $L_{h}(z, \vec{v})$ is independent of the choice of the orthonormalized system $f_{k}(z)$.

The orthonormalized basis consists of the functions

$$
f_{k}(z)=(k !)^{-\frac{1}{2}}\left(\frac{1}{h} \ldots\left(\frac{1}{h}-1+k\right)\right)^{\frac{1}{2}} z^{k} .
$$

Using these functions, we obtain that

$$
L_{h}(z, \bar{v})=(1-z \bar{v})^{-\frac{1}{h}} .
$$

Denote $L_{h}(z, \bar{v})$ through $\phi_{\bar{v}}(z)$, as the function $z$ at fix $\bar{v}$. 
It follows from (4.2) that $\phi_{v}(z) \in \mathscr{F}_{h}$ and for any function $f \in \mathscr{F}_{h}$

$$
\left(f, \phi_{\bar{z}}\right)=f(z) \text {. }
$$

The formula (4.4) means that a set of functions $\phi_{\bar{v}}$ forms the supercomplete system in $\mathscr{F}_{h}$. This system was examined before in [8].

2. Algebra of Covariant Symbols. Let $A$ be a bounded linear operator in $\mathscr{F}_{h}$. The covariant symbol of this operator constructed by means of the vectors $\phi_{\bar{z}}$ will be denoted through $A(z, \bar{z})$. Note that the function

$$
A(z, \bar{v})=\frac{\left(\hat{A} \phi_{\bar{v}}, \phi_{\bar{z}}\right)}{\left(\phi_{\bar{v}}, \phi_{\bar{z}}\right)}
$$

is analytically dependent upon $z$ and $\bar{v}$ coincides with $A(z, \vec{z})$ when $v=z$. Consequently, $A(z, \vec{z})$ is an analytic function of the variables $x=\operatorname{Re} z$, $y=\operatorname{Im} z$ and $A(z, \vec{v})$ is an analytic continuation of $A(z, \vec{z})$ in a complex region. Hence it follows that there is a one-to-one correspondence between the operators and their covariant symbols. In particular, if $A(z, \bar{z})=a=$ const then the corresponding operator $\hat{A}=a \hat{E}$ where $\hat{E}$ is a unit operator in $\mathscr{F}_{h}$.

Specificity of general formulas (3.10), (3.12), (3.15) gives

$$
(\hat{A} f)(z)=\left(\frac{1}{h}-1\right) \int A(z, \vec{v}) f(v)\left(\frac{1-v \bar{v}}{1-z \bar{v}}\right)^{\frac{1}{h}} d \mu(v, \bar{v})
$$

if $\hat{A}=\hat{A}_{1} \cdot \hat{A}_{2}$, then

$$
\begin{aligned}
& A(z, \vec{z})=\left(\frac{1}{h}-1\right) \int A_{1}(z, \vec{v}) A_{2}(v, \bar{z})\left[\frac{(1-z \bar{z})(1-v \bar{v})}{(1-z \bar{v})(1-v \bar{z})}\right]^{\frac{1}{h}} d \mu(v, \bar{v}), \\
& A(z, \bar{z})=\left(\frac{1}{h}-1\right) \int \AA(z, \bar{z})\left[\frac{(1-z \bar{z})(1-v \bar{v})}{(1-z \bar{v})(1-v \bar{z})}\right]^{\frac{1}{h}} d \mu(v, \vec{v}) .
\end{aligned}
$$

3. The Correspondence Principle. The operator $T_{h}$

$$
\left(T_{h} f\right)(z, \bar{z})=\left(\frac{1}{h}-1\right) \int f(v, \vec{v})\left[\frac{(1-z \bar{z})(1-v \bar{v})}{(1-z \bar{v})(1-v \bar{z})}\right]^{\frac{1}{h}} d \mu(v, \bar{v})
$$

is considered in the space of continuously differentiable functions in a unit circle. We find asymptotics of the function

$$
\varphi_{h}(z, \bar{z})=\left(T_{h} f\right)(z, \bar{z}) \text { for } h \rightarrow 0 .
$$

First we consider the case $z=0$ :

Note that

$$
\left(T_{h} f\right)(0,0)=\left(\frac{1}{h}-1\right) \int f(v, \vec{v})(1-v \vec{v})^{\frac{1}{h}} d \mu(v, \vec{v}) .
$$

$$
\left(\frac{1}{h}-1\right) \int(1-v \bar{v})^{\frac{1}{h}} d \mu(v, \bar{v})=\left(\frac{1}{h}-1\right) \int_{0}^{1}(1-x)^{\frac{1}{h}} d x=1 .
$$


Therefore,

$$
\left(T_{h} f\right)(0,0)=f(0,0)+\left(T_{h} f_{1}\right)(0,0) \quad \text { where } \quad f_{1}(v, \vec{v})=f(v, \vec{v})-f(0,0) .
$$

The function $f_{1}$, is continuous and $f_{1}(0,0)=0$. We consider the number $\varepsilon>0$ and partition the integral (4.9) substituting in it $f$ for $f_{1}$, in the sum:

$$
\begin{aligned}
\left(T_{h} f_{1}\right)(0,0)= & \left(\frac{1}{h}-1\right) \int_{v \bar{v}<\varepsilon} f_{1}(v, \vec{v})(1-v \bar{v})^{\frac{1}{h}} d \mu(v, \vec{v}) \\
& +\left(\frac{1}{h}-1\right) \int_{\varepsilon \leqq v \bar{v}<1} f(v, \vec{v})(1-v \bar{v})^{\frac{1}{h}} d \mu(v, \vec{v}) .
\end{aligned}
$$

Estimate of the 1-st integegral:

$$
\begin{aligned}
\left|\left(\frac{1}{h}-1\right) \int_{v \bar{v}<\varepsilon} f_{1}(1-v \bar{v})^{\frac{1}{h}} d \mu\right| & =\max _{v \bar{v}<\varepsilon}\left|f_{1}\right|\left(\frac{1}{h}-1\right) \int_{|v|<1}(1-v \bar{v})^{\frac{1}{h}} d \mu \\
& \leqq \max _{v \bar{v}<\varepsilon}\left|f_{1}\right| .
\end{aligned}
$$

Estimate of the 2-nd integral:

$$
\left|\left(\frac{1}{h}-1\right) \int_{\varepsilon \leqq v \bar{v}<1} f_{1}(1-v \bar{v})^{\frac{1}{h}} d \mu\right| \leqq(1-\varepsilon)^{\frac{1}{h}-2}\left(\frac{1}{h}-1\right) \int_{|v|<1}\left|f_{1}\right| \frac{d v d \bar{v}}{2 \pi} .
$$

Obviously when $\varepsilon>0$, then $\lim _{h \rightarrow 0}(1-\varepsilon)^{\frac{1}{h}-2}\left(\frac{1}{h}-1\right)=0$. Therefore, $\varlimsup_{h \rightarrow 0}\left|\left(T_{h} f_{1}\right)(0,0)\right| \leqq \max _{v \bar{v}<\varepsilon}\left|f_{1}\right|$ and due to continuity of $f_{1}, \lim _{\varepsilon \rightarrow 0} \varlimsup_{h \rightarrow 0}\left|T_{h} f_{1}\right|=0$. On the other hand, since $\varlimsup_{h \rightarrow 0}\left|T_{h} f_{1}\right|$ is independent of $\varepsilon$, hence it follows that $\overline{\lim }\left|\left(T_{h} f_{1}\right)(0,0)\right|=0$, consequently, there exists $\lim _{h \rightarrow 0}\left(T_{h} f_{1}\right)(0,0)$ and $\lim _{h \rightarrow 0}\left(T_{h} f_{1}\right)(0,0)=0$.

Turning again to the function $f$, we obtain the existence of $\lim _{h \rightarrow 0}\left(T_{h} f\right)(0,0)$ and the equality

$$
\lim _{h \rightarrow 0}\left(T_{h} f\right)(0,0)=f(0,0) .
$$

An arbitrary continuously differentiable function in $K$ can be written (ambigously) as

$$
f(v, \vec{v})=f(0,0)+v f_{1}(v, \vec{v})+\bar{v} f_{2}(v, \vec{v}),
$$

where $f_{1}, f_{2}-$ the continuous functions.

We note that for $h \rightarrow 0$

$$
\begin{aligned}
&\left(\frac{1}{h}-1\right) \int v f_{1}(1-v \bar{v})^{\frac{1}{h}-2} \frac{d v \wedge d \bar{v}}{2 \pi i}=-\int f_{1} \frac{\partial}{\partial \bar{v}}(1-v \bar{v})^{\frac{1}{h}-1} \frac{d v \wedge d \bar{v}}{2 \pi i} \\
&=\int \frac{\partial f_{1}}{\partial \bar{v}}(1-v \bar{v})^{\frac{1}{h}+1} d \mu(v, \bar{v})=\left.h \frac{\partial f_{1}}{\partial \bar{v}}\right|_{v=\bar{v}=0}+o(h)
\end{aligned}
$$


(the previous result has been applied to the function $\frac{\partial f_{1}}{\partial \bar{v}}$ ). Analogously

$$
\left(\frac{1}{h}-1\right) \int \bar{v} f_{2}(1-\bar{v} v)^{\frac{1}{h}} d \mu(v, \bar{v})=\left.h \frac{\partial f_{2}}{\partial v}\right|_{v=\bar{v}=0}+o(h) .
$$

Thus, finally,

$$
\begin{aligned}
\left(T_{h} f\right)(0,0)= & f(0,0)+\left.h\left(\frac{\partial f_{2}}{\partial \bar{v}}+\frac{\partial f_{2}}{\partial v}\right)\right|_{v=\bar{v}=0}+o(h)=f(0,0) \\
& +\left.h \frac{\partial^{2} f}{\partial v \partial \bar{v}}\right|_{v=\bar{v}=0}+o(h) .
\end{aligned}
$$

Proceed now to studying the Transformation (4.9) at arbitrary $z$. Change the variables

$$
v=\frac{w-z}{1-\bar{z} w} .
$$

The transformation $v \rightarrow w$ is a motion in the Lobachevskii plane (a reflection in some point). Therefore, the measured $d \mu$ is invariant under the change (4.12). Due to this change the integral (4.9) assumes the form

$$
\left(T_{h} f\right)(z, \bar{z})=\left(\frac{1}{h}-1\right) \int \phi(w, \bar{w})(1-w \bar{w})^{\frac{1}{h}} d \mu(w, \bar{w})
$$

where $\phi(w, \bar{w})=f\left(\frac{w-z}{1-\bar{z} w}, \frac{\bar{w}-\bar{z}}{1-z \bar{w}}\right)$.

We note that

$$
\left.\frac{\partial^{2} \phi}{\partial w \partial \bar{w}}\right|_{w=\bar{w}=0}=(1-z \bar{z})^{2} \frac{\partial^{2} f}{\partial z \partial \bar{z}}
$$

The operator $\Delta=(1-z \bar{z})^{2} \frac{\partial^{2}}{\partial z \partial \bar{z}}$ is the Laplace-Beltrami operator on the Lobachevskii plane.

Thus, using (4.11), we obtain an asymptotic decomposition for $T_{h} f$

$$
\left(T_{h} f\right)(z, \bar{z})=f(z, \bar{z})+h \Delta f(z, \bar{z})+o(h) .
$$

The correspondence principle follows from (4.14): putting $A_{1}(z, \bar{v}) A_{2}(v, \bar{z})$ $=f(v, \vec{v})$ in (4.7), we get

$$
\begin{aligned}
A(z, \bar{z})= & f(z, \bar{z})+h \Delta f+o(h)=A_{1}(z, \bar{z}) A_{2}(z, \bar{z}) \\
& +h(1-z \bar{z})^{2} \frac{\partial A_{1}}{\partial \bar{z}} \frac{\partial A_{2}}{\partial z}+o(h)
\end{aligned}
$$


Hence: $\lim _{h \rightarrow 0} A_{1} * A_{2}=A_{1}(z, \vec{z}) A_{2}(z, \bar{z})$ is the first requirement of the correspondence principle and $\lim _{h \rightarrow 0} \frac{1}{h}\left(A_{1} * A_{2}-A_{2} * A_{1}\right)=(1-z \bar{z})^{2}$ $\cdot\left(\frac{\partial A_{1}}{\partial \bar{z}} \frac{\partial A_{2}}{\partial z}-\frac{\partial A_{1}}{\partial z} \frac{\partial A_{2}}{\partial \bar{z}}\right)=i\left[A_{1}, A_{2}\right]$ is the second one.

4. The Operator $T_{h}$ Expressed in Terms of the Laplace-Beltrami Operator. The operator $T_{h}$ is permutable with the transformations $f(z, \vec{z}) \rightarrow f(g z, \bar{g} \vec{z})$ where $g$ is a motion in the Lobachevskii plane. From this property it follows that it is the function of the Laplace-Beltrami $\Delta$ operator.

Denote a kernel of the operator $T_{h}$ through $G_{h}(v, \bar{v} \mid z, \bar{z})$ and the transformation $\left(\tau_{z} f\right)(v, \vec{v})=f\left(\frac{z-v}{1-\bar{z} v}, \frac{\bar{z}-\bar{v}}{1-z \bar{v}}\right)$ through $\tau_{z}$. Note that

$$
G_{h}(v, \bar{v} \mid z, \bar{z})=\left(\tau_{z} G_{h}\right)(v, \bar{v} \mid 0,0) .
$$

(This feature of the function $G_{h}$ in the other notations has been used in the previous $n^{0}$.)

Using permutativity of the operator $\Delta$ with $T_{h}$ and $\tau_{z}$ and selfadjointness of $\Delta$, and $\tau_{z}$, we obtain:

$$
\begin{aligned}
\left(\Delta T_{h} f\right)(z, \bar{z}) & =\left(T_{h} \Delta f\right)(z, \bar{z})=\int(\Delta f)(v, \vec{v})\left(\tau_{z} G_{h}\right)(v, \bar{v} \mid 0,0) d \mu(v, \vec{v}) \\
& =\int f(v, \bar{v})\left(\tau_{z} \Delta_{v \bar{v}} G_{h}\right)(v, \bar{v} \mid 0,0) d \mu(v, \bar{v}) \\
& =\int\left(\tau_{z} f\right)(v, \vec{v})\left(\Delta_{v, \bar{v}} G_{h}\right)(v, \bar{v} \mid 0,0) d \mu(v, \bar{v}) .
\end{aligned}
$$

Further, after transformations, we obtain

$$
\begin{aligned}
\left(\Delta_{v, \bar{v}} G_{h}\right)(v, v \mid 0,0) & =\left(\frac{1}{h}-1\right)(1-v \bar{v})^{2} \frac{\partial^{2}}{\partial v \partial \bar{v}}(1-v \bar{v})^{\frac{1}{h}} \\
& =\frac{1}{h}\left(\frac{1}{h}-1\right)\left(G_{h}-G_{\frac{h}{h+1}}\right) .
\end{aligned}
$$

Consequently, the analogous relation is valid for the operators $T_{h}$, hence

$$
T_{h}=\left(1-\frac{\Delta}{\frac{1}{h}\left(\frac{1}{h}-1\right)}\right)^{-1} T_{\frac{h}{1+h}} .
$$

Iterating this relation we find, that

$$
T_{h}=\prod_{0}^{N} \frac{1}{1-h^{2} \frac{\Delta}{(1+n h)(1+(n-1) h)}} T \frac{h}{1+(N+1) h} .
$$

The first requirement of the correspondence principle means that $\lim _{h \rightarrow 0} T_{h}=E$ where $E$ is a unit operator. Therefore, proceeding to the limit for $N \rightarrow \infty$ in (4.16), we obtain the final expression of the operator 
$T_{h}$ through $\Delta$

$$
T_{h}=\prod_{n=0}^{\infty} \frac{1}{1-h^{2} \frac{\Delta}{(1+n h)(1+(n-1) h}} .
$$

5. Representations of the Group of Motions of the Lobachevskii Plane in the Space $\mathscr{F}_{h}$. Denote this group through $G$.

Consider the transformation in $A_{h}$

$$
\left(\tau_{g} A\right)(z, \bar{z})=A(g z, \overline{g z}), \quad g \in G .
$$

It has been mentioned above that the transformation (4.18) is the automorphism of the algebra $A_{h}$. As is known, all automorphisms of the algebra of bounded operators in a Hilbert space are internal. Therefore, the bounded operator which generates automorphism (4.18) exists in $\mathscr{F}_{h}$ $\left(\hat{U}_{g} \hat{A} \hat{U}_{g}^{-1} \phi_{\bar{z}}, \phi_{\bar{z}}\right)\left(\phi_{\bar{z}}, \phi_{\bar{z}}\right)^{-1}=\left(\hat{A} \phi_{g z}, \phi_{\overline{g z}}\right)\left(\phi_{g z}, \phi_{\overline{g z}}\right)^{-1}=A(g z, \overline{g z})$.

The operator $\hat{U}_{g}$ is defined up to arbitrary complex multiplier. Since the Transformation (4.18) transfers the real functions in to the real, the transformation $\hat{A} \rightarrow \hat{U}_{g} \hat{A} \hat{U}_{g}^{-1}$ transfers self-adjoint operators into self- adjoint. Hence it follows that $\hat{U}_{g}$ differs from the unitary operator by the multiplier only. Thus, one can choose $\hat{U}_{g}$ unitary, the indefinite multiplier equals 1 in modulus.

In what follows we consider this condition to be satisfied. It is obvious that the correspondence $g \rightarrow \hat{U}_{g}$ is the projective representation of $G$. We show that it is irreducible. Let $\hat{A}$ be the bounded operator permutable with all $\hat{U}_{g}$. It follows from (4.19) that in this case $A(g z, \overline{g z})$ is independent of $g$. Due to the fact that $G$ is transitive, $A(z, \bar{z})=a=$ const. It follows from a one-to-one correspondence between the operators and the covariant symbols that $\hat{A}=a \hat{E}$, where $\hat{E}$ is a unit operator in $\mathscr{F}_{h}$. Consequently, the representation is irreducible.

Show the explicit form of the operators $\hat{U}_{g}$. Let $g^{-1}=\left(\begin{array}{ll}a & b \\ \bar{b} & \bar{a}\end{array}\right)$ then

$$
\left(\hat{U}_{g} f\right)(z)=\theta f\left(\frac{a z+b}{\bar{b} z+\bar{a}}\right)(\bar{b} z+\bar{a})^{-h}, \quad|\theta|=1 .
$$

The unitarity of Transformations (4.20) is verified directly.

The property (4.19) follows from

$$
\begin{aligned}
\left(\hat{U}_{g} \phi_{\bar{v}}\right)(z) & =\theta\left(1-\bar{v} \frac{a z+b}{\bar{b} v+\bar{a}}\right)^{-h}(\bar{a}+\bar{b} z)^{-h}=\theta(\bar{b} z+a-\bar{v}(a z+b))^{-h} \\
& =\theta\left(1-\frac{a \bar{v}-\bar{b}}{-b \bar{v}+a} z\right)^{-h}(\bar{a}-b \bar{v})^{-h}=\theta(\bar{a}-b \bar{v})^{-h} \phi_{g^{-1} v}(z)
\end{aligned}
$$

In conclusion we indicate the covariant symbol $U_{g}(z, \bar{z})$ of the operator $\hat{U}_{g}$. Combining (4.1) and (4.4) we get:

$$
\begin{aligned}
\left(\hat{U}_{g} \phi_{\bar{z}}, \phi_{\bar{z}}\right) & =\theta(\bar{a}-b \bar{z})^{-h}\left(\phi_{\overline{g^{-1} z}}, \phi_{\bar{z}}\right)=\theta(\bar{a}-b \bar{z})^{-h} \phi_{\overline{g^{-1} z}}(z) \\
& =\theta(\bar{a}-a z \bar{z}-b \bar{z}+\bar{b} z)^{-h} .
\end{aligned}
$$


Hence

$$
U_{g}(z, \bar{z})=\theta\left(\hat{U}_{g} \phi_{\bar{z}}, \phi_{\bar{z}}\right)\left(\phi_{\bar{z}}, \phi_{\bar{z}}\right)^{-1}=\theta\left(\frac{1-z \bar{z}}{\bar{a}-a z \bar{z}-b \bar{z}+\bar{b} z}\right)^{h} .
$$

6. Quantization by Means of Reflections (Analog of the Weyl Quantization). Let $g(v, \vec{v})$ being reflection in the point $v$

$$
g(v, \bar{v}) z=\frac{-(1+v \bar{v}) z+2 v}{-2 \bar{v} z+1+v \bar{v}}, \quad g=\frac{1}{1-v \bar{v}}\left(\begin{array}{ll}
-(1+v \bar{v}) & 2 v \\
-2 \bar{v} & 1+v \bar{v}
\end{array}\right) .
$$

The operator $\hat{U}_{g}$ for $g=g(v, \vec{v})$ will be denoted via $\hat{U}_{v, \bar{v}}$. According to the general formula (4.22) the covariant symbol of the operator $\hat{U}_{v, \bar{v}}$ up to the multiplier $\theta,|\theta|=1$, has the form

$$
U_{h}(v, \bar{v} \mid z, \bar{z})=\left[\frac{(1-z \bar{z})(1-v \bar{v})}{(1-z \bar{v})(1-v \bar{z})}\right]^{h} \frac{1}{\left[1+\frac{z-v}{1-\bar{z} v} \frac{\bar{z}-\bar{v}}{1-z \bar{v}}\right]^{h}} .
$$

We fix the operator $\hat{U}_{z, \bar{z}}$ so that its covariant symbol has exactly the form of (4.24).

The function

$$
\mathscr{A}(z, \bar{z})=\operatorname{Sp}\left(\hat{A} \hat{U}_{z, \bar{z}}\right)
$$

will be called the Weyl covariant symbol of the operator $\hat{A}$. The function $\mathscr{A}$, by means of which the operator $\hat{A}$ is represented by the integral

$$
\hat{A}=\left(\frac{1}{h}-1\right) \int \stackrel{\mathscr{A}}{(z, \bar{z})} \hat{U}_{z, \bar{z}} d \mu(z, \bar{z})
$$

will be called the Weyl contravariant symbol of the operator.

The connection between the symbols $\AA$ and $\mathscr{A}$ as well between $\mathscr{A}$ and $A$ is given by

$$
\begin{aligned}
\mathscr{A}(z, \bar{z}) & =\left(S_{h} \stackrel{\AA}{ }\right)(z, \bar{z}), \\
A(z, \bar{z}) & =\left(S_{h} \mathscr{A}\right)(z, \bar{z}),
\end{aligned}
$$

where

$$
\left(S_{h} f\right)(z, \vec{z})=\left(\frac{1}{h}-1\right) \int f(v, \vec{v}) U_{h}(v, \bar{v} \mid z, \vec{z}) d \mu(v, \vec{v})
$$

[the formula (4.27) follows from (3.15) and from the symmetry

$$
\left.U_{h}(v, \bar{v} \mid z, \bar{z})=U_{h}(z, \bar{z} \mid v, \bar{v})\right] .
$$

To express the symbols $\mathscr{A}$ and $\mathscr{A}$ one via another, we need the operator $S_{h}$ as the function of the Laplace-Beltrami operator $\Delta$. This is possible due to permutativity of $S_{h}$ and the operators (4.18) $\tau_{g}$.

Performing transformations, analogous to (4.15), we obtain

$$
\left(\Delta S_{h} f\right)(z, \bar{z})=\left(\frac{1}{h}-1\right) \int\left(\tau_{z} f\right)(v, \vec{v})\left(\Delta_{v, \bar{v}} U_{h}\right)(v, \bar{v} \mid 0,0) d \mu(v, \vec{v}) .
$$


By means of elementary transformations we see that

$$
\begin{aligned}
\left(\Delta_{v, \bar{v}} U_{h}\right)(v, \bar{v} \mid 0,0) & =(1-v \bar{v})^{2} \frac{\partial^{2}}{\partial v \partial \bar{v}}\left(\frac{1-v \bar{v}}{1+v \bar{v}}\right)^{\frac{1}{h}} \\
& =\frac{1}{h}\left[\left(\frac{1}{h}-1\right)\left(\frac{1-v \bar{v}}{1+v \bar{v}}\right)^{\frac{1}{h}}-\left(\frac{1}{h}+1\right)\left(\frac{1-v \bar{v}}{1+v \bar{v}}\right)^{\frac{1}{h}+2}\right] .
\end{aligned}
$$

Hence

$$
\Delta S_{h}=\frac{1}{h}\left(\frac{1}{h}-1\right)\left(S_{h}-S_{\frac{2 h}{1+2 h}}\right), \quad S_{h}=\left(1-h^{2} \frac{\Delta}{1-h}\right)^{-1} S_{\frac{2 h}{1+2 h}} .
$$

Iterating this relation, we obtain

$$
S_{h}=\prod_{0}^{n-1}\left(1-h^{2} \frac{\Delta}{(1+2 k h)(1+(2 k-1) h)}\right)^{-1} S_{\frac{2 h}{1+2 n h}} .
$$

Applying the calculations of $n^{0} 3$ to the operators $S_{h}$, we have that $\lim _{h \rightarrow 0} S_{h}=E$. Therefore, passing in (4.29) to the limit $n \rightarrow \infty$ we receive

$$
S_{h}=\prod_{0}^{\infty}\left(1-h^{2} \frac{\Delta}{(1+2 k h)(1+(2 k-1) h)}\right)^{-1} \text {. }
$$

Comparing (4.17) and (4.30), we get

where

$$
T_{h}=S_{h} S_{h}^{\prime}
$$

$$
S_{h}^{\prime}=\prod_{0}^{\infty}\left[1-h^{2} \frac{\Delta}{(1+(2 k+1) h)(1+2 k h)}\right]^{-1} \text {. }
$$

It follows from (4.31) and (4.8)

$$
\stackrel{\circ}{\mathscr{A}}=S_{h}^{\prime} \stackrel{\circ}{A}=S_{h}^{\prime} S_{h}^{-1} \mathscr{A} \text {. }
$$

\section{§ 5. Quantization on a Sphere}

When the number $n=\frac{1}{h}$ is integer, the theory, relating to a sphere, copies in detail the theory refering to the Lobachevskii plane. Let us consider such a case. At first sight the condition of integrability of $\frac{1}{h}$ seems exstravagant. However, as it will be shown in $\S 6$, quantization on a sphere is, under some natural subsidiary conditions, unique up to equivalence. Thus, we are free from considering non-integers $\frac{1}{h}$.

The main formulas will be given without deduction. Assume radius of a sphere $r=\frac{1}{2}$. A sphere is looked upon as the widened complex plane. In this case the invariant measure on a sphere reads $d \mu(z, \bar{z})$ $=(1+z \bar{z})^{-2} \frac{d z \wedge d \bar{z}}{2 \pi i}[\operatorname{see}(1.7)]$. 
1. The space $\mathscr{F}_{h}$ consists of holomorphic functions $f(z)$ with scalar product

$$
(f, g)=\left(\frac{1}{h}+1\right) \int f(z) \overline{q(x)}(1+z \bar{z})^{-\frac{1}{h}} d \mu(z, \bar{z}) .
$$

We remark that if $f(z) \in \mathscr{F}_{h}$, then $f(z)$ is a polynomial of the power no higher then $\frac{1}{h}$ : in all rest cases the integral, which defines $(f, f)$, diverges.

Thus, $\operatorname{dim} \mathscr{F}_{h}=1+\frac{1}{h}$.

The vectors

$$
\phi_{\bar{v}}(z)=(1+z \bar{v})^{\frac{1}{h}}
$$

form a supercomplete system in $\mathscr{F}_{h}$. For any $f \in \mathscr{F}_{h}$ following formula is valid

$$
\left(f, \phi_{\bar{v}}\right)=f(v) .
$$

We note that (5.2) becomes meaningless under noninteger $\frac{1}{h}$ in this case $\phi_{\bar{v}}(z)$ is a multiple valued function and separation of ambiguous branch is impossible ${ }^{5}$.

2. Algebra of Covariant Symbols. The covariant symbol $A(z, \bar{z})$ of the operator $\hat{A}$ is the value of the function when $v=z$

$$
A(z, \bar{v})=\frac{\left(\hat{A} \phi_{\bar{v}}, \phi_{\bar{z}}\right)}{\left(\phi_{\bar{v}}, \phi_{\bar{z}}\right)} .
$$

The Function (5.4) is an analytic continuation of $A(z, \bar{z})$ therefore, there is a one-to-one correspondence between the covariant symbols and operators.

The operator action onto the vector

$$
(\hat{A} f)(z)=\left(\frac{1}{h}+1\right) \int A(z, \vec{v}) f(v)\left(\frac{1+z \bar{v}}{1+v \bar{v}}\right)^{\frac{1}{h}} d \mu(v, \bar{v}) .
$$

The product of the operators; if $\hat{A}=\hat{A}_{1} \cdot \hat{A}_{2}$, then

$$
A(z, \vec{v})=\left(\frac{1}{h}+1\right) \int A_{1}(z, \vec{v}) A_{2}(v, \bar{z})\left[\frac{(1+z \bar{v})(1+v \bar{z})}{(1+z \bar{z})(1+v \bar{v})}\right]^{\frac{1}{h}} d \mu(v, \bar{v}) .
$$

Connection between co- and contravariant symbols

$A(z, \vec{z})=\left(T_{h} \AA\right)(z, \bar{z})=\left(\frac{1}{h}+1\right) \int \AA(v, \vec{v})\left[\frac{(1+z \vec{v})(1+v \vec{z})}{(1+v \vec{v})(1+z \bar{z})}\right]^{\frac{1}{h}} d \mu(v, \vec{v})$.

The expression of the operator $T_{h}$ through the Laplace-Beltrami operator:

$$
T_{h}=\prod_{1}^{\infty}\left(1+h^{2} \frac{\Delta}{(1+n h)(1+(n+1) h)}\right) .
$$

The asymptotic formula (4.11) is valid for the operator $T_{h}$. The correspondence principle follows from this formula, as in the case of the Lobachevskii plane.

3. Connection with Representations. The motions of a sphere produce automorphisms $\tau_{g}$ of the algebra $A_{h}$ according to (4.18). Denote the

\footnotetext{
${ }^{5}$ The System (5.2) (in other coordinates) is the well-known system of Bloch coherent
} states. See for example [9]. 
projective representation of the group $S O(3)$ which is connected with automorphisms $\tau_{g}$ by (4.19), through $U_{g}$.

The group $S O(3)$ acts onto a sphere according to

The operator $\hat{U}_{g}$ is defined by

$$
z \rightarrow g^{-1} z=\frac{a z+b}{-b \bar{z}+\bar{a}} .
$$

$$
\left(\hat{U}_{g} f\right)(z)=f\left(\frac{a z+b}{-\bar{b} z+\bar{a}}\right)(-\bar{b} z+\bar{a})^{\frac{1}{h}} .
$$

(Under the odd $\frac{1}{h}$ the representation $\hat{U}_{g}$ is two-valued.) It is well-known from the representation theory, that the whole set of projective representations $S O(3)$ is exhausted up to equivalence by the representations $\hat{U}_{g}$.

\section{§ 6. Problems of Uniqueness}

1. Additional Definitions. Let $\mathfrak{U}_{1}$ and $\mathfrak{A}_{2}$ be the quantizations of the same classical mechanics. The quantization $\mathfrak{U}_{1}$ will be named subquantization $\mathfrak{U}_{2}$ designated as $\mathfrak{U}_{1} \subset \mathfrak{U}_{2}$ if there exists the admissible monomorphism $\psi: \mathfrak{U}_{1} \rightarrow \mathfrak{U}_{2}$.

Quantisation $\mathfrak{A}$ is colled maximum, if from $\mathfrak{A} \subset \mathfrak{U}_{1}$ follows $\mathfrak{A}=\mathfrak{U}_{1}$. Let $\mathfrak{A}$ be a special quantization of the mechanics $(\mathfrak{M}, \omega)$ natural with respect to some category $\mathscr{K}$ which involves the group $G$ of motions of the manifold $\mathfrak{M}$.

As it has been already mentioned, in this case the shifts generate automorphisms of the algebra $A_{h}$ by

$$
\left(\tau_{g} f\right)(x)=f(g x) .
$$

The quantization $\mathfrak{A}$ will be effective if there is no natural isomorphism (i.e. permutable with all $\tau_{g}$ ) between the algebras $A_{h_{1}}, A_{h_{2}}$ for $h_{1} \neq h_{2}$.

The quantization will be irreducible if the algebras allow the exact irreducible representations by the bounded operators in a Hilbert space.

The quantization will be named $w^{*}$ quantization if the algebras $A_{h}$ are the $w^{*}$ algebras.

In particular, for the irreducible $w^{*}$ quantization the algebras $A_{h}$ are isomorphic with respect to complete algebras of bounded operators in a Hilbert space.

2. General Consideration. Consider a set $\tilde{M}$ of the algebras $A$, consisting of functions on a homogeneous manifold $\mathfrak{M}$ with the group of motions $G$ and having the following features:

i) The algebra $A$ is isomorphic to the algebra of bounded operators in a Hilbert space.

ii) The shifts $\left(\tau_{g} f\right)(x)=f(g x)$ are the automorphisms of the algebra $A$.

iii) A unit of the algebra $A$ is the function $f_{0}(x) \equiv 1$.

The algebras $A_{1}, A_{2} \in \tilde{M}$ will be called naturally isomorphic if there exists an isomorphism between them, which is permutable with the automorphism $\tau_{g}$. 
A set of all classes of the algebras $A$, naturally isomorphic in pairs, will be denoted through $M$.

A set of all irreducible projective representations of the group $G$ will be further denoted via $\tilde{T}$ and via $T$ a set of classes of unitarily equivalent projective representations of $G$. Let us construct the monomorphic mapping $M \rightarrow T$.

We fix $A \in \tilde{M}$. Denote the isomorphic $A$ algebra of bounded operators in a Hilbert space through $L$, and through $\varphi$ the isomorphism $A \rightarrow L$. Let $\sigma_{g}=\varphi \tau_{g} \varphi^{-1}$ be the automorphism $L$. Since all the automorphisms $L$ are internal, there exists the bounded operator $\hat{U}_{g}$, which is defined up to a numerical multiplier $\lambda$ and produces automorphism $\sigma_{g}$ :

$$
\sigma_{g} \hat{f}=\hat{U}_{g} \hat{f} \hat{U}_{g}^{-1} \text {. }
$$

Due to the fact that $\sigma_{g}$ is an * automorphism, it transfers the hermitian operators into hermitian. Hence it follows that $\hat{U}_{g}$ differs from unitary operator by the multiplier only. Thus, one can consider $\hat{U}_{g}$ to be the unitary operator, an indefinite multiplier $\lambda$ to be such, that $|\lambda|=1$.

The operators $\hat{U}_{g}$ perform the unitary projective representation of the group $G$.

Prove the fact that $\hat{U}_{g}$ is irreducible. Let $\hat{f}_{0} \in L$ be permutable with $\hat{U}_{g}$ and $f_{0}(x)=\varphi^{-1} \hat{f}_{0} \in A$. Due to (6.2)

$$
f_{0}(g x)=\left(\tau_{g} f_{0}\right)(x)=\varphi^{-1} \sigma_{g} \hat{f}_{0}=\varphi^{-1}\left(\hat{U}_{g} \hat{f}_{0} \hat{U}_{g}^{-1}\right)=\varphi^{-1} \hat{f}_{0}=f_{0}(x) .
$$

It follows from transitivity of actions of the group $G$ onto $\mathfrak{M}$ that $f_{0}(x)=$ const $=f_{0}$. In virtue of the feature iii) it means that $\hat{f}_{0}=f_{0} E$ where $E$ is a unit operator in $\mathscr{H}$. Therefore, the irreducible projective representation $\hat{U}_{g} \in \tilde{T}$ of the group $G$ is confronted with each algebra $A \in \tilde{M}$.

We show that the algebras $A_{1}, A_{2} \in \tilde{M}$ are naturally isomorphic if and only if the representations $\hat{U}_{g}^{(1)}$ and $\hat{U}_{g}^{(2)}$ corresponding to them, are unitarily equivalent.

We give the indices $i, i=1,2$ to the objects, refering to the algebra $A_{i}$. Let the representations $\hat{U}_{g}^{(i)}$ be unitarily equivalent and $V: \mathscr{H}_{1} \rightarrow \mathscr{H}_{2}$ be isomorphism of Hilbert spaces, which performs this equivalence: $V \hat{U}_{g}^{(1)}=\hat{U}_{g}^{(2)} V$. We construct the isomorphisms $L_{1} \rightarrow L_{2}$ and $A_{1} \rightarrow A_{2}$ by means of $V$

$$
\hat{f} \rightarrow V \hat{f} V^{-1}, \quad f(x) \rightarrow \varphi_{2}^{-1}\left(V\left(\varphi_{1} f\right) V^{-1}\right)=\psi f .
$$

The isomorphism (6.3) $f \rightarrow \psi f$ is natural:

$$
\begin{aligned}
\psi \tau_{g}^{(1)} f & =\varphi_{2}^{-1}\left(V \hat{U}_{g}^{(1)} \varphi_{1} f\left(\hat{U}_{g}^{(1)}\right)^{-1} V^{-1}\right) \\
& =\varphi_{2}^{-1}\left(\hat{U}_{g}^{(2)} V \varphi_{1} f V^{-1}\left(\hat{U}_{g}^{(2)}\right)^{-1}\right)=\tau_{g}^{(2)} \psi f
\end{aligned}
$$

Vice verse, let the algebras $A_{1}, A_{2}$ be naturally isomorphic and $\psi: A_{1} \rightarrow A_{2}$ be isomorphism. In this case $\chi=\varphi_{2} \psi \varphi_{1}^{-1}$ is isomorphism of the algebras $L_{1}$ and $L_{2}$. Since $L_{1}$ and $L_{2}$ are the complete operator algebras, there exists the isomorphism $V: \mathscr{H}_{1} \rightarrow \mathscr{H}_{2}$ which generates $\chi: \chi \hat{f}=V \hat{f} V^{-1}$, $\hat{f} \in L_{1}$. The naturalness $\psi$ means the equality $\psi \tau_{g}^{(1)}=\tau_{g}^{(2)} \psi$. It follows from this that $\chi \sigma_{g}^{(1)}=\sigma_{g}^{(2)} \chi$ where $\sigma_{g}^{(i)}$ is the automorphism of $L_{i}$, such as (6.2). 
In more detail:

Hence

$$
\begin{aligned}
& V \hat{U}_{g}^{(1)} \hat{f}\left(\hat{U}_{g}^{(1)}\right)^{-1} V^{-1}=\hat{U}_{g}^{(2)} V \hat{f} V^{-1}\left(\hat{U}_{g}^{(2)}\right)^{-1} \\
& V^{-1}\left(\hat{U}_{g}^{(2)}\right)^{-1} V \hat{U}_{g}^{(1)} \hat{f}=\hat{f} V^{-1}\left(\hat{U}_{g}^{(2)}\right)^{-1} V \hat{U}_{g}^{(1)}
\end{aligned}
$$

As (6.5) is valid at any $\hat{f} \in L_{1}$, then $V^{-1}\left(\hat{U}_{g}^{(2)}\right)^{-1} V \hat{U}_{g}^{(1)}=\lambda E$, consequently, $\hat{U}_{g}^{(1)}=\lambda V^{-1} \hat{U}_{g}^{(2)} V$. The equality obtained denotes unitary equivalence of the projective representations $\hat{U}_{g}^{(1)}$ and $\hat{U}_{g}^{(2)}$.

Thus, the correspondence $A \rightarrow \tilde{U}_{g}$ where $\hat{U}_{g}$ is irreducible projective representation of the group $G$, which is defined by (6.2), is a monomorphic mapping of the classes $M \rightarrow T$.

3. Description of Quantizations on a Sphere. We show that quantization on a sphere, described in $\S 5$, is unique maximum effective irreducible $w^{*}$ quantization up to natural equivalence. Let $\mathfrak{A}$ be such a quantization, $A_{h}$ the corresponding algebras, $\tilde{M}, M, \tilde{T}$, $T$ is a specialization of the objects, introduced in No. 2, when $\mathfrak{M}=S_{2}$ and $G=S O(3)$ is a group of motions. The algebras $A_{h}$ have the features i)-iii), i.e. $A_{h} \in \tilde{M}$. Since there is no natural isomorphism between $A_{h}$ at different $h$, classes of the algebras $A \in \tilde{M}$, naturally isomorphic to $A_{h}$, are different at different $h$. We denote a set of classes involves $A_{h}$, through $M_{\mathscr{N}}$. The quantization $\mathfrak{A}$ is completely defined by the set $M_{\mathfrak{U}}$, and if $\mathfrak{A}_{1} \subset \mathfrak{A}_{2}$ then $M_{\mathfrak{A}_{1}} \subset M_{\mathfrak{U}_{2}}$. Denote the quantization, described in $\S 5$, through $\mathfrak{A}_{0}$. To prove our assertion it suffices to check that $M_{\mathfrak{Q}_{0}}=M$. For this purpose we use the monomorphism constructed in the previous section. Let $T_{\mathfrak{U}_{0}}$ and $T_{M}$ be the images of $M_{\mathfrak{Y}_{0}}$ and $M$ under this monomorphism. The following inclusions are obvious: $T_{\mathscr{V}_{0}} \subset T_{M} \subset T$. It has been mentioned in $\S 5$ that the representations $\hat{U}_{g}$ defined by (6.2), are exhausted up to equivalence of projective representations of the group $S O(3)$. Consequently, $T_{\mathfrak{I}_{0}}=T$ and, therefore $T_{\mathfrak{I}_{0}}=T_{M}, M_{\mathfrak{I}_{0}}=M$.

We have not succeeded in describing analogously the quantization on the Lobachevskii plane, since for the Lobachevskii plane only the inclusion $T_{\mathfrak{P}_{0}} \subset T$ is valid, but not the equality $T_{\mathfrak{I}_{0}}=T$.

\section{References}

1. Weyl, H.: Gruppentheorie und Quantenmechanik. Leipzig, 1931

2. Berezin, F.A.: Funktzionalnyi analiz i ego prilozheniya, 1, 1-14 (1967) (in Russian)

3. Berezin, F.A.: Dokl. Akad. Nauk SSSR, 211 (No. 6) (1973) (in Russian)

4. Berezin, F.A.: Quantization on bounded complex symmetric. Izv. Akad. Nauk SSSR, ser. mat., in press (1974) (in Russian)

5. Berezin, F.A.: Izv. Akad. Nauk, ser. mat. 36 (No. 5) (1972); (in Russian). English translation: USSR Izv. 6 (No. 5) (1972)

6. Bergmann, S.: The Kernel function and conformal mapping. A.M.S. Publ. 1950

7. Berezin,F.A.: Quantization. Izv. Akad. Nauk SSSR, ser. mat. in press (1974) (in Russian)

8. Monastyirski, M.I., Perelomov, A.M.: Dokl. Akad. Nauk SSSR 207, 1303-1305 (1972)

9. Lieb, E.H.: Commun. math. Phys. 31, 327-340 (1973)

Communicated by R. Haag

F. A. Berezin

Moscow State University

Moscow B-234, USSR 